\title{
DISTÚRBIOS DA CONDUTA ALIMENTAR: ANOREXIA E BULIMIA NERVOSAS
}

\author{
DISORDERS OF EATING BEHAVIOUR: ANOREXIA NERVOSA AND BULIMIA
}

Rosane Pilot Pessa Ribeiro1; Paulo César Monteiro dos Santos² \& José Ernesto dos Santos ${ }^{3}$

\begin{abstract}
${ }^{1}$ Docente do Departamento de Enfermagem Materno-Infantil e Saúde Pública da Escola de Enfermagem de Ribeirão Preto-USP, ${ }^{2}$ Médico Psiquiatra, Doutor em Saúde Mental pela Faculdade de Medicina de Ribeirão Preto-USP; ${ }^{3}$ Docente do Departamento de Clínica Médica da Faculdade de Medicina de Ribeirão Preto-USP.

Correspondência: Prof.Dr. José Ernesto dos Santos - Departamento de Clínica Médica da Faculdade de Medicina de Ribeirão Preto da Universidade de São Paulo - Campus Universitário - CEP: 14048-900 - Ribeirão Preto - SP; FAX: (016) 633-1144; E-mail: jedsanto@fmrp.usp.br
\end{abstract}

RIBEIRO RPP; SANTOS PCM \& SANTOS JE. Distúrbios da conduta alimentar: anorexia e bulimia nervosas. Medicina, Ribeirão Preto, 31: 45-53, jan./mar. 1998.

RESUMO: A anorexia e a bulimia nervosas são distúrbios da conduta alimentar, que se caracterizam pela abstenção voluntária dos alimentos e pela ingestão compulsiva, seguida de vômitos, respectivamente. Compreendem, sindromicamente, uma série de distúrbios psicológicos e comportamentais, associados a alterações somáticas, características de desnutrição do tipo marasmática, principalmente nos casos de anorexia nervosa. Esses quadros têm sido descritos predominantemente em adolescentes do sexo feminino, de classe sócio-econômica média e alta, quase sempre em países desenvolvidos.

Neste trabalho, são apresentadas características do diagnóstico clínico, antropométrico e laboratorial de sessenta e oito pacientes, atendidos no Ambulatório de Distúrbios da Conduta Alimentar e do Peso, do Hospital das Clínicas da Faculdade de Medicina de Ribeirão Preto-USP, no período entre 1982 e 1997.

Considerando que essas síndromes têm elevada prevalência e são acompanhadas de alta morbidade, é preconizado o melhor conhecimento de suas manifestações clínicas, tanto no meio médico como no populacional, a fim de que o diagnóstico possa ser definido o mais precocemente possível, facilitando o tratamento e favorecendo o prognóstico.

UNITERMOS: Distúrbios Alimentares. Anorexia Nervosa. Bulimia. Distúrbios Nutricionais. Nutrição do Adolescente.

"It (anorexia nervosa) rarely if even, affects poor people and has not been described in underdeveloped countries" Hilde Brush, "The golden cage: The enigma of anorexia nervosa". Vintake Books, New York, pg VIII, 1979.

A anorexia e a bulimia nervosas são distúrbios da conduta alimentar que vêm apresentando elevada prevalência, não só nos países desenvolvidos, onde subsistem as características econômicas e sócio-culturais para o seu desencadeamento, como, também, nos países do terceiro mundo ${ }^{(1,2,3)}$.

Ao mesmo tempo, devido ao seu quadro clínico intrigante e de mau prognóstico, estes transtornos têm merecido a atenção crescente dos profissionais da área da saúde e da sociedade. 


\section{ANOREXIA NERVOSA}

Descrita, inicialmente, por Morton, em 1694(4), como consumpção nervosa, a anorexia nervosa afeta, principalmente, jovens adolescentes, do sexo feminino, que voluntariamente reduzem a ingestão alimentar por um medo mórbido de engordar, com perda progressiva e desejada de peso, chegando a graus extremos de caquexia, inanição e até à morte. Como síndrome psicossomática, compreende alterações somáticas, importantes, caracterizadas pela desnutrição proteicocalórica e amenorréia, além de distúrbios psicológicos como depressão, fobia, compulsões, preocupação obsessiva com os alimentos, distorção de imagem corporal, entre outros ${ }^{(5)}$.

A etiopatogenia inclui um conjunto de fatores de ordem biológica, psicológica, familiar e sócio-cultural, e não, como no passado se tentou atribuir, fatores isolados ${ }^{(6)}$. $\mathrm{O}$ amplo espectro de distúrbios psicológicos e comportamentais que integram a síndrome sugere que a sua origem seja psicogênica, embora de natureza obscura. Dentro dessa linha, admite-se que a anorexia nervosa ocorra, com grande freqüência, em famílias de alto nível social, que apresentam padrões inadequados ou destrutivos de relacionamento interpessoal, caracterizados por uma aparente harmonia, mas por poderosos conflitos subjacentes. Valorizam mais o sucesso e o jogo de aparências do que a verdadeira realização pessoal dos seus membros. A jovem pré-anoréxica se adapta muito bem às expectativas parentais, pois, pela sua inteligência, obediência, perfeccionismo e dedicação aos estudos, colabora para manter a aparente harmonia da vida familiar, mesmo em detrimento da sua própria personalidade em formação ${ }^{(7)}$.

Porém, com o início da adolescência e o conjunto de mudanças de esfera biológica, psicológica e social, esta jovem, aparentemente bem ajustada, é invadida por sentimentos de ineficiência, desamparo e descontrole sobre o seu corpo e sua vida. Assim, a busca desesperada pela magreza representa o esforço para readquirir o controle e a harmonia perdidos ${ }^{(8)}$.

A medida em que a doença progride, muitas vezes, iniciando com dietas para emagrecer, aliadas a programas exaustivos de exercícios físicos, o emagrecimento tende a se acentuar. E é justamente pelo quadro de desnutrição que os familiares buscam tratamento médico, muitas vezes, com resistência por parte do paciente.

A incidência mundial é estimada em 1:100.000 ${ }^{(9)}$, porém, se considerarmos apenas as mulheres jovens e brancas, de países desenvolvidos, esta taxa se eleva para 1:200 ${ }^{(10)}$. Há evidências de que a incidência esteja aumentando nas últimas décadas, em países como os Estados Unidos e os da Europa ${ }^{(11,12)}$, além de ser oportuno considerar os grupos de risco, ou seja, pessoas preocupadas com o peso e a forma física (modelos, bailarinas, aeromoças e artistas) no aumento da incidência.

O diagnóstico da anorexia nervosa é feito segundo os critérios estabelecidos pelo DSM-IV ${ }^{(13)}$ (Tabela I), sendo fundamental afastar doenças de caráter tumoral, hormonal, gastrointestinal, além de certos quadros psiquiátricos, como a depressão e a esquizofrenia (Tabela II).

Tabela I - Critérios diagnósticos para anorexia nervosa (307-1) - DSM IV

1 - Recusa em manter o peso corporal, ideal ou acima do peso mínimo para idade e altura.

2 - Medo intenso de ganhar peso ou tornar-se obeso, mesmo se abaixo do peso ideal.

3 - Distúrbios de imagem corporal.

4 - Amenorréia em mulheres pós-menarca (ausência de, pelo menos, três ciclos menstruais consecutivos).

Subtipos:

- Restritivo: restrição dietética.

- Compulsivo/Purgativo: ingestão excessiva/vômitos, laxativos, diuréticos.

\section{Tabela II - Diagnóstico diferencial para anorexia nervosa - DSM IV}

1 - Doenças gastrointestinais e consumptivas (AIDS, câncer).

2 - Síndrome da artéria mesentérica, superior.

3 - Depressão e esquizofrenia.

\section{2 - BULIMIA NERVOSA}

A bulimia nervosa foi caracterizada como síndrome distinta, a partir da década de 80, pois, anteriormente, figurava apenas como sintoma coadjuvante à anorexia nervosa. Russel, em $1985^{(14)}$, considera o fato, devido à supreendente mudança que a anorexia nervosa vinha sofrendo nas suas formas de depressão/ou apresentação clínica, em provável resposta às modificações ocorridas no contexto sócio-cultural. 
Por definição, esta síndrome se caracteriza por surtos de ingestão maciça dos alimentos, seguidos por comportamentos compensatórios de eliminação, por vômitos, uso abusivo de laxantes, diuréticos e atividade física excessiva. Esses acessos incontroláveis de ingestão alimentar ocasionam sentimentos de culpa, vergonha e depressão pela falta de controle ${ }^{(5)}$.

Comumente, a anorexia nervosa incide em jovens adolescentes, que apresentam medo intenso de engordar e distúrbios de imagem corporal. Porém, o aspecto comportamental sugere que elas se apresentem mais extrovertidas, com caráter histérico, vida sexual ativa e, muitas vezes, dependentes de álcool e/ou drogas.

A etiopatogenia da bulimia nervosa inclui, também, com grande freqüência, conflitos familiares, história de abuso sexual na infância e questões sobre o desenvolvimento de feminilidade, ocasionando assim, dificuldade de controle de impulsos, depressão crônica, exagerados sentimentos de culpa e intolerância à frustração e à ansiedade.

As manifestações físicas, apresentadas por pacientes com bulimia, não são tão comprometedoras quanto ao estado nutricional, ao contrário do que é visto na anorexia nervosa. Vários distúrbios gastrointestinais, alguns deles sérios, e, mesmo, potencialmente fatais, são descritos na literatura, como dilatação gástrica aguda, inclusive com possibilidade de ruptura, hipertrofia de parótidas, desgaste do esmalte dentário, esofagite, esvaziamento gástrico, anormal e síndrome do cólon irritável. A hipopotassemia representa uma séria complicação, relativamente freqüente, em virtude da associação dos vômitos crônicos ao uso de diuréticos e/ou laxativos ${ }^{(13)}$.

Para o diagnóstico da bulimia nervosa, utilizam-se os critérios estabelecidos pelo DSM-IV(13) (Tabela III). Faz-se necessário distingui-la da anorexia nervosa, já que ela pode, também, acontecer como sintoma coadjuvante da mesma, ou de forma isolada, em subtipos de doença afetiva, esquizofrenia e obesidade, quando ocorrem episódios noturnos de voracidade, com usuários de psicofármacos (particularmente antidepressivos) e deficientes mentais. A Tabela IV mostra o diagnóstico diferencial dessa síndrome.

O tratamento dos transtornos alimentares é, quase sempre, desafiador e complexo, visto que se depara com um quadro de confusão, perplexidade e, mesmo, de franco desespero do paciente e de seus familiares. Assim, várias modalidades terapêuticas específicas são necessárias e desenvolvidas por equipe multiprofissional.
Entre elas, destacam-se a terapia nutricional, baseada na orientação e reeducação alimentar, a psicofarmacoterapia, a psicoterapia individual e a de grupo familiar ${ }^{(15)}$.

$\mathrm{Na}$ literatura, poucos trabalhos foram publicados, mostrando casuísticas e resultados do tratamento de distúrbios alimentares no Brasil. Santos et al., ${ }^{(16)}$ em 1986, fez uma revisão de doze casos de anorexia nervosa, atendidos no primeiro serviço criado para esse fim, em 1982, o Ambulatório de Distúrbios da Conduta Alimentar e do Peso, do Hospital das Clínicas da Faculdade de Medicina de Ribeirão Preto - USP (HCFMRP-USP). Mais tarde, em 1990(5), os mesmos publicam extensa revisão do assunto, abordando aspectos que incluem desde o reconhecimento das síndromes até o prognóstico das mesmas, com dados clínicos, antropométricos e laboratoriais de pacientes atendidas pelo mesmo serviço.

\section{Tabela III - Critérios diagnósticos para bulimia ner- vosa (307.51) - DSM IV}

1 - Episódios recorrentes de compulsão alimentar, que pode ser caracterizada por:

a) comer, em período de duas horas, grande quantidade de alimentos;

b) sentimento de perda do controle alimentar, durante o episódio.

2 - Comportamento compensatório para prevenir o ganho de peso: vômitos auto-induzidos, abuso de laxativos, diuréticos, enemas ou outras drogas, jejum ou exercícios excessivos.

3 - A compulsão alimentar e comportamentos compensatórios ocorrem duas vezes/semana, por, pelo menos, três meses.

4 - Preocupação excessiva com a forma corporal e o peso.

5 - O distúrbio não ocorre exclusivamente durante episódios de Anorexia Nervosa.

Subtipos:

- Purgativo: vômitos ou abuso de laxativos, diuréticos e enemas.

- Não purgativo: jejum ou exercícios excessivos.

Tabela IV - Diagnóstico diferencial para bulimia nervosa - DSM IV

1 - Anorexia Nervosa - tipo compulsivo/purgativo.

2 - Depressão com características atípicas.

3 - Distúrbios de personalidade. 
Com tais trabalhos, verifica-se que a existência de distúrbios alimentares, em países em desenvolvimento, é notória, e, talvez, subestime-se a sua incidência devido a diversos fatores, entre eles a negação radical da síndrome por parte do paciente e de sua família, retardando ou excluindo, perigosamente, a busca de assistência médica. Some a isso, ainda, o fato de que os médicos e a equipe multiprofissional ainda não estejam, em geral, suficientemente sensibilizados para o reconhecimento precoce das mesmas e preparados para tratá-la corretamente.

O fato é que, entre uma coisa e outra ou, na soma das duas, perde-se um tempo crucial, em que se poderia ter feito o diagnóstico, senão precoce, pelo menos, oportuno. E, perda de tempo, nesses casos, como em tantas outras situações médicas, críticas, geralmente, significa maior dificuldade em tratar, cronicidade, deterioração e, mesmo, morte.

\section{A EXPERIÊNCIA DA DIVISÃO DE NUTROLOGIA DA FMRP-USP - ANÁLISE DE SESSENTA E OITO CASOS EM QUINZE ANOS}

Este estudo clínico foi realizado pela análise das observações e dos registros dos prontuários hospitalares dos sessenta e oito pacientes, atendidos no Ambulatório de Distúrbios da Conduta Alimentar e do Peso - HCFMRP-USP, desde o seu início, em 1982, até outubro de 1997.

Esses casos, em função da sua urgência nutricional, foram espontaneamente encaminhados, principalmente por médicos e serviços da cidade e região, na maioria das vezes, sem diagnóstico e, nos demais, sob suspeita clínica ou, em poucos casos, com diagnóstico estabelecido. Vale acrescentar que, raramente, houve iniciativa pessoal na busca de atendimento médico, o que só aconteceu sob constrangimento familiar, explicando, de certo modo, o freqüente retardo no início do tratamento.

\subsection{A primeira entrevista}

Devido a esses fatores, a primeira entrevista foi conduzida de maneira cuidadosa, a fim de amenizar o clima de conflito entre os familiares e o paciente, e adquirir a confiança dos mesmos. Nesse sentido, alguns princípios foram observados: a) evitar exortações, reprimendas ou ameaças, que apenas reproduzem situações já bastante conhecidas e muito bem manipuladas pelo paciente, dentro do contexto familiar; b) evitar qualquer sugestão que induza o paciente e a família a se sentirem culpados pelo que está acontecendo; a idéia a ser passada é que existe um sério problema de saúde, cuja solução é de responsabilidade de todos; c) adotar uma atitude compreensiva e afetuosa, mas firme e desenvolta - própria de quem conhece o problema que se apresenta, para tratá-lo, ou pelo menos, dar-lhe o encaminhamento devido.

Adotou-se, nesse primeiro contato, a seqüência assim resumida: a) constatação da queixa principal e motivo da consulta, na presença de todos - pacientes e familiares; b) entrevista com o paciente, para realização de avaliação clínica, incluindo exame físico e antropométrico geral (peso, altura); c) entrevista com a família, para averigüar informações dadas pelo paciente e até complementação das mesmas; d) entrevista com o paciente e família, para relatar o diagnóstico e dar noções gerais a respeito da síndrome e suas consequiências - particularmente sobre o quadro de desnutrição e seus efeitos sobre o estado mental e comportamento - das indicações terapêuticas e do prognóstico. Na sequiência, pedem-se exames laboratoriais de rotina, para avaliação do estado nutricional, e inicia-se o tratamento, sob a forma de hospitalização ou regime ambulatorial.

\subsubsection{Tratamento ambulatorial e critérios de internação}

Nos casos de início recente do quadro, em que não houve ainda perda significativa de peso ou, inversamente, nos casos crônicos com preservação relativa do estado nutricional, a melhor indicação é a assistência ambulatorial. Ainda assim, costuma-se alertar o paciente e família quanto à possibilidade de hospitalização, se houver algum retrocesso, complicações médicas ou, mesmo, falta de recuperação satisfatória de peso. Essa advertência antecipada permite dividir a responsabilidade por uma futura internação e torná-la mais aceitável, caso venha a se tornar efetivamente necessária. Caso isso aconteça, adotaram-se os seguintes critérios para sua indicação: a) externa e rápida perda de peso (30\% ou mais em relação ao habitual ou desejável, dentro de um período de três meses); b) alteração de sinais vitais (hipotensão postural, bradicardia, hipotermia); c) distúrbios eletrolíticos, principalmente hipopotassemia (geralmente, resultante do abuso de diuréticos/laxativos ou vômitos recorrentes) e desidratação; d) infecção intercorrente em pacientes severamente caquéticos; e) tentativa de suicídio ou tendência suicida e reações psicóticas. 
Além dessas situações, a internação pode ser indicada também a partir de critérios psicossociais, como deterioração do quadro por grave distúrbio familiar ou isolamento social, e psicoterapêuticos, como carência de motivação para o seguimento ambulatorial.

$\mathrm{Na}$ abordagem desses distúrbios, a decisão de hospitalizar ou tratar ambulatorialmente é particularmente crítica e, qualquer que seja ela, deve fazer parte de um amplo e bem estruturado plano de tratamento. Para o paciente internado, o Ambulatório de Distúrbios de Conduta Alimentar conta com equipe multiprofissional, composta por médico clínico, psiquiatra, nutricionista, além do assistente social e enfermeiro, que desenvolvem as modalidades terapêuticas propostas.

\subsection{Quadro clínico, laboratorial e antropomé- trico}

Durante esses quinze anos de funcionamento, sessenta e oito pacientes foram atendidos no Ambulatório de Distúrbios da Conduta Alimentar e do Peso - HCFMRP-USP. Desses, quarenta e sete pacientes receberam o diagnóstico de anorexia nervosa, tipo restritivo (AN-R), dezessete pacientes com tipo compulsivo/purgativo ou bulímico (AN-B) e quatro apresentavam bulimia nervosa $(\mathrm{BN})$. Conforme mostra a Tabela V, a maioria era do sexo feminino $(\mathrm{n}=$ 64) com idade variando de onze a cinquienta e dois anos, conforme o diagnóstico. Vale notar que os casos de bulimia nervosa $(\mathrm{BN})$ eram com pacientes de idade mais avançada que a daqueles com anorexia nervosa, do tipo restritivo (AN-R).
Em relação aos parâmetros antropométricos, os pacientes com AN-R apresentaram os índices mais baixos, caracterizando quadro de desnutrição de grau III, enquanto, nos que tinham AN-B, essa deficiência era menos pronunciada. Os pacientes com $\mathrm{BN}$, do ponto de vista nutricional, eram eutróficos (Tabela VI).

Concomitante à avaliação antropométrica, quando do primeiro atendimento, realizou-se a avaliação bioquímica desses pacientes. Geralmente, poucas alterações foram detectadas além de níveis séricos de ferro no limite inferior da normalidade e hiperbetacarotenemia. O padrão protéico (demonstrado pela albumina e proteínas totais) foi normal em todos os tipos de distúrbios alimentares, conforme mostra a Tabela VII.

A amenorréia foi outro sinal investigado durante a avaliação clínica, visto que é um dos itens dos critérios diagnósticos para essas síndromes. Dos sessenta e quatro pacientes do sexo feminino, nove (14\%) não haviam apresentado menarca na época do primeiro atendimento. Das mulheres em idade fértil $(\mathrm{n}=55)$, quarenta $(72,7 \%)$ estavam em amenorréia há mais de três meses.

Devido ao risco de dano ósseo, em todas as pacientes, que se encontram por período longo sem menstruar, realizamos densitometria óssea, a partir do ano de 1996. Onze pacientes foram submetidas a tal avaliação. Observou-se que quatro delas $(36,3 \%)$ apresentam quadro de osteopenia em coluna lombar e sete pacientes $(63,6 \%)$ já desenvolveram osteoporose, tanto em coluna lombar como no fêmur. Esses resultados demonstram a importância dessa avaliação e a necessidade de indicar esquema de reposição hormonal, como vem acontecendo nessa amostra.

O comportamento alimentar, apresentado pelos pacientes atendidos nesse período, foi bastante típico, em relação ao descrito na literatura. Nos casos de anorexia nervosa, notou-se restrição intensa da ingestão alimentar (principalmente dos alimentos mais calóricos, ricos em carboidratos e gorduras), diminuição do número de refeições, caracterizando, muita vezes, um padrão alimentar típico de dietas vegetarianas, com exclusão de carnes vermelhas e 
TABELA VI - Avaliação antropométrica dos pacientes com distúrbios alimentares - HCFMRP-USP (X \pm SD)

\begin{tabular}{lcccc} 
& AN-R & AN-B & BN & Valores de referência \\
\hline IMC $\left(\mathrm{Kg} / \mathrm{m}^{2}\right)$ & $15,0 \pm 3,6$ & $17,7 \pm 3,9$ & $20,8 \pm 2,5$ & $19-24$ \\
Perda de peso $(\%)$ & $26,5 \pm 11,2$ & $23,4 \pm 13,4$ & - & - \\
PCT $(\mathrm{mm})$ & $5,2 \pm 3,1$ & $10,9 \pm 7,5$ & $16,5 \pm 6,6$ & 16,5 \\
CB $(\mathrm{cm})$ & $18,1 \pm 4,6$ & $21,4 \pm 4,5$ & $26,1 \pm 2,0$ & 28,5 \\
CMB $(\mathrm{cm})$ & $15,5 \pm 3,1$ & $17,9 \pm 2,7$ & $20,8 \pm 1,4$ & 23,2 \\
\hline
\end{tabular}

IMC = índice de massa corporal $\left(P(\mathrm{~kg}) /\right.$ altura $\left.^{2}(\mathrm{~m})\right)$.

$\mathrm{PCT}=$ prega cutânea triciptal.

$\mathrm{CB}=$ circunferência braquial.

$\mathrm{CMB}=$ circunferência muscular do braço $(\mathrm{CMB}=\mathrm{CB}-\pi \times \mathrm{PCT})$.

Tabela VII - Avaliação bioquímica dos pacientes com distúrbios alimentares - HCFMRP-USP $(X \pm S D)$

\begin{tabular}{lccc}
\hline & AN-R & AN-B & BN \\
\hline Albumina $(\mathrm{g} / \mathrm{dl})$ & $4,6 \pm 0,7$ & $4,9 \pm 0,7$ & $5,1 \pm 1,0$ \\
Proteínas totais $(\mathrm{g} / \mathrm{dl})$ & $7,4 \pm 1,0$ & $7,5 \pm 0,7$ & $7,6 \pm 1,0$ \\
Ferro $(\mu \mathrm{g} / \mathrm{dl})$ & $89 \pm 37$ & $86 \pm 20$ & $85 \pm 52$ \\
TIBC $(\mu \mathrm{g} / \mathrm{dl})$ & $288 \pm 98$ & $285 \pm 69$ & $389 \pm 106$ \\
$\beta$ - caroteno $(\mu \mathrm{g} / \mathrm{dl})$ & $189 \pm 107$ & $220 \pm 127$ & $148 \pm 8,2$ \\
Vitamina C $(\mathrm{mg} / \mathrm{d})$ & $0,32 \pm 0,15$ & $0,39 \pm 0,1$ & $0,39 \pm 0,19$ \\
\hline
\end{tabular}

TIBC = capacidade total de ligação do ferro.

predomínio de alimentos reguladores, como frutas e vegetais. Além disso, o uso de alimentos dietéticos foi freqüente (refrigerantes, adoçantes) e até a ingestão de água mostrou-se bastante prejudicada. Outros comportamentos, de caráter obsessivo, em relação à alimentação, foram detectados: 1) cortar os alimentos em pedaços bem pequenos e remexe-los no fundo do prato antes de ingeri-los; 2) mastigar lentamente pequena quantidade de alimento e depois, cuspir; 3) esconder os alimentos ou jogar fora, dizendo para a família que a ingestão dos mesmos aconteceu; 4) evitar comer na presença de outras pessoas; 5) observar, curiosamente, as refeições alheias e insistir para que os outros comam de tudo; 6) fiscalizar a cozinha e a despensa, fazendo compras no supermercado; 7) colecionar receitas culinárias, preparar pratos e demonstrar singular interesse por Nutrição.

Nos casos de bulimia nervosa, a ingestão alimentar, nos momentos de compulsão, era muito alta, prejudicando, até, o cálculo calórico, por dificuldade dos pacientes precisarem as quantidades dos alimentos. Além disso, os episódios de vômitos, autoprovocados ou espontâneos, estavam sempre presentes, às vezes concomitantes ao uso de laxantes e diuréticos. 


\subsection{Abordagem terapêutica}

\subsubsection{Evolução clínica}

Conforme comentado anteriormente, o tratamento se desenvolveu, de preferência, a nível ambulatorial, salvo em alguns casos, em que os pacientes se encontravam gravemente desnutridos e com complicações clínicas de alto risco.

Ao todo, vinte e sete pacientes necessitaram de internação hospitalar (39,7\%), a maioria deles $(92,5 \%)$ portadores de anorexia nervosa.

O período de internação variou bastante, mas, na maioria dos casos, quase nunca foi inferior a quatro semanas. Alguns pacientes $(n=5)$ tiveram mais de um período de internação.

A análise da evolução ponderal mostrou-se, na maioria das vezes, bastante positiva. A média de recuperação de peso foi de $5,1 \mathrm{Kg}$, nos casos de anorexia nervosa. Aqueles pacientes com bulimia nervosa $(\mathrm{n}=2)$ chegaram a perder peso (em média $2,2 \mathrm{Kg}$ ), devido ao controle alimentar, conseqüente à diminuição dos episódios, ingestão compulsiva e vômitos. Esse resultado é explicado pelo fato de estarem esses pacientes sempre entre o limite normal e superior de seu peso.

Durante a internação hospitalar, procurou-se adotar a via oral como preferencial, para oferecer os nutrientes necessários para a recuperação. No entanto, alguns pacientes com anorexia nervosa apresentaram dificuldade em alcançar a ingestão mínima, recomendada, por mais que ela fosse individualizada com as suas preferências alimentares. Nessas situações, o suporte nutricional foi indicado para prover o aporte de calorias e nutrientes, sendo pela sonda enteral o de primeira escolha, em dez pacientes. Optou-se por fórmulas poliméricas, administradas, de preferência, por bomba de infusão, no período noturno, para que, durante o dia, as refeições pudessem ser ingeridas.

O tratamento da anorexia nervosa e bulimia tende a ser árduo, prolongado e de resultados duvidosos e, muitas vezes, insatisfatórios. Isso se deve, em parte, às condições inerentes à doença que, na sua complexidade e rebeldia, representa um desafio a qualquer esforço terapêutico. Por outro lado, existem os mecanismos de negação, acionados pelos pacientes e seus familiares, no sentido de negar a gravidade, quando não, a própria existência do problema. Com isso, freqüentemente, não existe a mínima cooperação para o auxílio e a possibilidade de sucesso.

\subsection{Análise dos resultados}

Nos resultados apresentados, observou-se que as características da casuística, obtidas ao longo desses quinze anos, mostraram-se em concordância com dados encontrados na literatura mundial, ou seja, a maioria dos pacientes era do sexo feminino, prevalecendo os adolescentes, sendo mais comuns os casos de anorexia nervosa, do tipo restritivo. Conseqüentemente, deparou-se com quadros de extremo emagrecimento, sem, contudo, se encontrarem alterações bioquímicas importantes. Concluímos ser a desnutrição desses pacientes, freqüentemente, do tipo marásmica, com grande adaptação orgânica à diminuição da oferta calórica e concomitante aumento da atividade física, como forma adicional de provocar a perda de peso e adquirir a tão desejada magreza. Dados semelhantes a esta análise realizada por Santos ${ }^{(17)}$ vêm a corroborar os indicativos antropométricos e bioquímicos, característicos dessas síndromes.

Algumas vezes, a recuperação nutricional não foi obtida a nível de ambulatório, e a indicação de internação hospitalar foi necessária, para reverter as complicações clínicas da anorexia nervosa (desidratação, desnutrição grave) e da bulimia (desequilíbrio hidroeletrolítico, ingestão compulsiva e maciça dos alimentos, seguida de vômitos).

A evolução clínica, durante o seguimento, foi variável, com alta taxa de abandono do tratamento $(\mathrm{n}=39 ; 57,3 \%)$ após alguns atendimentos, principalmente naqueles casos em que o estado geral do paciente não era grave o suficiente para permitir a adesão imediata ao serviço. Dois casos de anorexia nervosa $(2,9 \%)$ evoluíram para óbito, em regime de internação. Atualmente, cerca de vinte pacientes estão em acompanhamento, alguns devido à cronicidade das doenças, outros que se encontram no início do tratamento, com boa evolução, e vários casos em quase recuperação completa.

Na literatura, poucos dados são encontrados sobre o prognóstico dessas síndromes devido, principalmente, a dificuldades metodológicas. Nemiah ${ }^{(18)}$ identificou, na sua amostra, $70 \%$ de cura ou melhora clínica dos seus casos, $22 \%$ de cronicidade e $8 \%$ de mortalidade, este último item bem maior do que o encontrado nesta casuística. Ainda sobre mortalidade, existe relato de porcentagem ainda maior $(20 \%)$ na Suécia, segundo Theander ${ }^{(19)}$.

Esses dados só tendem a reforçar a importância dos esforços terapêuticos, que devem sempre se basear em programas multidisciplinares, que promovam a reabilitação nutricional com base na reeducação 
alimentar, acompanhamento clínico, psicológico, comportamental e familiar, que visam abranger todos os intrigantes aspectos somáticos, psicológicos, familiares e sociais que se apresentam nos casos dos distúrbios alimentares.

Devido ao aumento da incidência, nos países desenvolvidos do mundo ocidental, nota-se grande preocupação dos cientistas em aperfeiçoar os métodos diagnósticos e terapêuticos que possam resultar na detecção de fatores de risco em grandes segmentos populacionais e no desenvolvimento de programas comunitários preventivos.

No nosso meio, apesar de não existirem dados concretos, podemos considerar, diante da experiência obtida e aqui mostrada, que haja subestimação da presença dos distúrbios alimentares, principalmente nos grandes centros urbanos e regiões mais desenvolvidas do país. Tal fato, associado à alta morbidade e mortalidade já detectada, exige imediata divulgação do quadro clínico dessas síndromes, nos meios de comunica- ção de massa, preparo e sensibilização dos médicos para o diagnóstico, diminuição da negação dos sintomas por parte do paciente e familiares, favorecendo, assim, a busca por assistência médica e, finalmente, formação de equipes multidisciplinares que promovam, o quanto antes, tratamento específico, a fim de serem evitadas a cronicidade e a morte.

\section{AGRADECIMENTO}

Os autores agradecem a todos os profissionais que participaram da assistência aos pacientes atendidos e colaboraram com a revisão dos dados aqui apresentados, em especial a Dra. Silviane Vianna, Dra. Maria Cristiane Rafael de Farias, Dr. Fernando B. Chueire, Dr. Dênis Eduardo Bertini Bó, Dr. Evaldo dos Santos, Dra. Ingrid Dick de Paula, Prof. Dr. Júlio Sérgio Marchini, enfermeira Maria do Rosário Del Lama Unamuno, nutricionista Milene Goretti A. Ferronato e funcionários da Unidade Metabólica - HCFMRP-USP.

RIBEIRO RPP; SANTOS PCM \& SANTOS JE. Disorders of eating behavior: Anorexia nervosa and bulimia. Medicina, Ribeirão Preto, 31: 45-53, jan./march 1998.

ABSTRACT: Anorexia nervosa and bulimia are eating disorders characterized by voluntary abstinence from food and by compulsive ingestion followed by vomiting, respectively. They syndromically consist of a series of psychological and behavioral disorders associated with somatic changes characteristic of malnutrition of the marasmus type, especially in cases of anorexia nervosa. These disorders have been predominantly reported for adolescent girls of middle to upper socioeconomic class, and almost always in developed countries.

In the present study we report the characteristics of the clinical, anthropometric and laboratory diagnosis of 68 patients seen at the Outpatient Clinic for Disorders of Eating Behavior and of Weight of the University Hospital, Faculty of Medicine of Ribeirão Preto, USP, from 1982 to 1997.

Considering that these syndromes are highly prevalent and are accompanied by high morbidity, a better understanding of their clinical manifestations is recommended both at the medical and populational level, so that a diagnosis may be made as early as possible, facilitating treatment and favoring prognosis.

UNITERMS: Eating Disorders. Anorexia, Nervosa. Bulimia. Nutrition Disorders. Adolescent Nutrition.

\section{REFERÊNCIAS BIBLIOGRÁFICAS}

1 - HOEK HW. Review of the epidemiological studies of eating disorders. Int Rev Psychiatry 5: 61-74, 1993.

2 - LUCAS AR et al. 50-year trends in the incidence of anorexia nervosa in Rochester, Minnesota: a population - based-study. Am J Psychiatry 148: 917-922, 1991.

3 - OYEWUMI LK \& KAZARIAN SS. Abnormal eating attitudes among a group of nigerian youths: II Anorexic behaviour. East Afr Med J 69: 667-669, 1992.
4 - MORTON R. Phthisiologia: or a treatise of consumption. Smith and Walford, London, p. 4-10, 1694.

5 - SANTOS PCM; PESSA RP \& DOS SANTOS JE. Como diagnosticar e tratar anorexia nervosa e bulimia. Rev Bras Med 47: 155-169, 1990.

6 - SIMMONDS M. Ueber embolische prozesse in der hyphophysis. Arch Pathol Anat 217: 226-239, 1914.

7 - BRUCH HB. Perceptual and conceptual disturbances in anorexia nervosa. Psychosom Medicine 24: 187-194, 1962. 
8 - CRISP AH. Anorexia nervosa. Hosp Med 713-718, May 1967.

9 - CRISP AH; PALMER RL \& KALWAY RS. How comum is anorexia nervosa? A prevalence study. Br J Psychiatry 128: 549-554, 1976.

10 - HALMI KA. Anorexia nervosa: demographic and clinical features in 94 cases. Psychosom Med 36: 18-24, 1974.

11 - HERZOG DB \& COPELAND PM. Eating disorders. N Engl J Med 313: 295-303, 1985.

12 - WILLI J \& GROSSMAN S. Epidemiology of anorexia nervosa in a defined region of Switzerland. Am J Psychiatry 140: 564-561, 1983.

13 - AMERICAN PSYCHIATRIC ASSOCIATION. Diagnostic and statistical manual of mental disorders. $4^{\text {th }}$. ed. Washington, 1994

14 - RUSSEL GFM. The changing nature of anorexia nervosa: an introduction to the conference. J Psychiatr Res 19: 101-109, 1985.
15 - GARNER DM et al. Psychoeducational principles in the treatment of bulimia and anorexia nervosa. In: GARNER DM \& GARFINKEL PE, eds. Anorexia nervosa and bulimia. Guilford Press, New York, p.513-572, 1985.

16 - SANTOS PCM et al. Anorexia nervosa: uma revisão clínica de 12 casos. Rev Paul Med 104: 240-246, 1986.

17 - SANTOS PCM. Ingestão alimentar e estado nutricional de pacientes com anorexia nervosa. Dissertação de Mestrado, Faculdade de Medicina de Ribeirão Preto da USP Ribeirão Preto, p. 66-73, 1995.

18 - NEMIAH J.C. Anorexia nervosa. Am J Dig Dis 3: 249-274 1958.

19 - THEANDER S. Research on outcome and prognosis of anorexia nervosa and some results from a Swedish long term study. Int J Eat Dis 2: 167-174, 1983.

Recebido para publicação em 30/01/98

Aprovado para publicação em 25/02/98 